\title{
HYDRODYNAMIC APPROACH TO IMPLOSION OF TARGET PLASMA BY LASER HEATING
}

Keishiro NIU and Takashi YABE

Department of Physical Engineering

Tokyo Institute of Technology

Ohokayama, Meguro-ku, Tokyo, Japan

\$ิ1. Introduction

So far calculations of implosion of a target pellet exposed to a laser light have been carried out in the United States ${ }^{1-5}$ and in other countries. In order to give some theoretical informations to the groups which are performing experiments in Japan ${ }^{7-8}$, we are now making the same kind of calculations. Here we would like to report some results and make several serious points clear in order to improve the thermalization and the compression of ions in the target plasma.

\section{ริ2. Fundamental Equations}

The fundamental equations governing the implosion are the following six spherically symmetric hydrodynamic equations.

The continuity equation : $\partial p / \partial t+1 / r^{2} \cdot \partial \rho u r^{2} / \partial r=0$.

The equation of motion :

$$
\frac{\partial u}{\partial t}+u \frac{\partial u}{\partial r}=-\frac{1}{\rho} \frac{\partial}{\partial r}(p+q)-\frac{4}{3 \rho r^{2}} \frac{\partial}{\partial r}\left(r^{2} \mu \frac{\partial u}{\partial r}\right)-\frac{4 u}{3 \rho} \frac{\partial}{\partial r}\left(\frac{\mu}{r}\right)-\frac{4 \mu u}{3 \rho r^{2}} .
$$

The reaction equation : $\left.\partial Y / \partial t+u \partial Y / \partial r=n(1-Y)^{2}<\sigma V\right\rangle / 4$.

$$
\dot{i} Y=\left(n_{\alpha}+n_{n}\right) /\left(n_{D}+n_{T}+n_{\alpha}+n_{n}\right) \cdot j
$$

The equation for the laser power intensity: $1 / r^{2} \cdot \partial r^{2} P_{L} / \partial r+k_{a} P_{L}=0$.

The energy equation for the ion :

$$
\partial T_{i} / \partial t+u \partial T_{i} / \partial r=-2 m_{j}\left(p_{i}+q\right) / 3 k \rho r^{2} \cdot \partial r^{2} u / \partial r-\left(T_{i}-T_{e}\right) \tau e_{i}+W_{i}+Q_{i} \text {. }
$$

The energy equation for the electron :

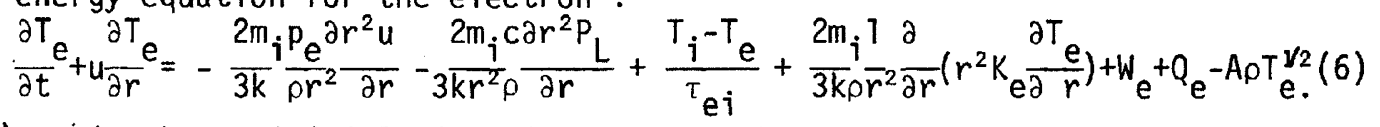

In eq. (2), $q$ is the artificial viscosity term to which the expressions

$q=c(\partial u / \partial r)^{2}$ if $\partial u / \partial r<0, \quad q=0$ if $\partial u / \partial r>0$

are e mp loyed $d^{9}$. For the classical absorption in the under-dense region, the absorption coefficient $K_{a}$ in eq. (4) is given by ${ }^{10}$

$$
k_{a}=\lambda^{2} n^{2} / 5 \times 10^{27}\left(T_{e} / 1.16 \times 10^{4}\right)^{3 / 2}\left(1-n / n_{c}\right)^{1 / 2} \text {, }
$$

and for the anomalous absorption at and near the critical density regions, we use the following expression ${ }^{11}$,

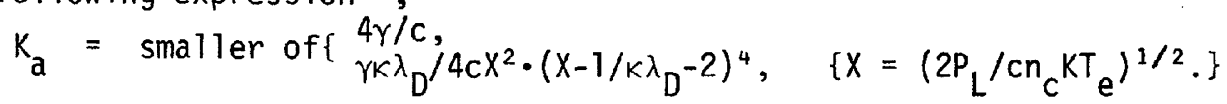

where $\gamma$ is the imaginary part of $\omega$ which satisfies the following equation for parametric instabilities 12 ,

$$
\omega^{2}+2 i \omega \Gamma_{1}-\omega_{1}^{2}=2 \pi \kappa^{2} e^{2} \dot{P}_{L}\left\{1 /\left(\omega+\delta+i \Gamma_{2}\right)-1 /\left(\omega-\delta+i \Gamma_{2}\right)\right\} / \mathrm{em}_{i} \mathrm{~m}_{\mathrm{e}} \mathrm{e}_{2} \text {. }
$$

In eqs. (5) and (6), $W_{i, e}$ shows the energy which comes from reactions, and $Q_{j, e}$ is the dissipation energy caused by the viscosity.

After eqs. (1)-(6) are transformed to Lagrangian forms, they are approximated by the difference equations to be carried out numerical calculations.

§3. Numerical Results

The numerical model of the plasma density of the target pellet is shown in Fig.l. At first, the laser power intensity $P_{L}$ is assumed to be constant in time outside the pellet. A result is given in Fig.2. Results are checked by using the energy conservation. The errors involved in calculations are less than $5 \%$. Figure 3 shows that the maximum values of $n, T_{j}, T_{e}$ realized at the pellet center in the course of time and the total number $\mathrm{N}_{\text {eu }}$ of neutron yields versus the laser power density $P_{L}$. It is turned out that the most efficient compression and the most 
efficient heating of ions occur at different values of $P_{L}$. Thus, there is the optimum value of $P L$ which leads us to the maximum nuclear reaction.

After the target plasma is irradiated by a laser light, a part of the plasma converges to the center of the pellet and another part expands into the vacuum. The ratio of the energy $E_{1}$ of the expanding part to the energy $E_{2}$ of the converging part is from 7 to 20 for various $P_{L}$. The majority of the laser energy absorbed in the target plasma escapes from the pellet and does not contribute directly to the fusion reaction. We can show from the plane one-dimensional similar solutions ${ }^{13}$ that $E_{1} / E_{2}=\left(m_{2} n_{2} / m_{1} n_{1}\right)^{1 / 2}$, if the energy $E=E_{1}+E_{2}$ is released at an instant on the boundary of the two different substances 1 and 2, provided that the boundary does not move. Therefore, we emphasize that the implosion is efficiently performed if the outside part of the critical density of the target plasma is replaced by a substance with a heavier particle mass. In the following calculations, we choose $\mathrm{m}_{i 1}=10 \mathrm{~m}_{\mathrm{i}}$.

The aspect of implosion changes according to the dependence of the laser power on time. We assume that dependence of the laser power $E^{2}$ on time is given by $\dot{E}=\dot{E}_{0}(1-t / \tau)^{-2}$

where $E_{0}$ and $\tau$ are constant. The fusion energy $E_{\text {out }}$ emitted from the pellet is plotted against $E_{0}$ in fig.4. For large pellets, larger $E_{0}$ is efficient for increasing Eout. Figure 5 indicates that the optimum value of $\tau$ exists for the realization of the maximum $\mathrm{N}_{\mathrm{eu}}$. Figure6shows the fusion energy Eout versus the pellet size $r_{0}$ when $E_{0}$ and $\tau$ take the optimum values, respectively.

\$4. Stability of Implosion

In order to realize the thermonuclear fusion reaction in the target pellet by a laser heating, the implosion must take place in a stable manner. In $\$ 2$, we assume that the motion is spherically symmetric. Now we check the stabilities of our results reported in the preceding section.

Here we consider the three-dimensional motion of the target plasma ${ }^{14}$. The fundamental equations in the polar coordinate system $r, \theta$ and $\phi$ are, $\dot{\partial} \rho / \partial t+\operatorname{div} \rho \vec{v}=0$,

$\partial \vec{v} / \partial t+\operatorname{grad} v^{2} / 2-\vec{v} \times \operatorname{rot} \vec{v}=-1 / \rho \cdot \operatorname{grad} p$,

$3 k \rho / 2 m_{i} \cdot D T e / D t=-\rho p_{e} \cdot D(1 / \rho) / D t+\nabla \cdot\left(K_{e} \nabla T_{e}\right)+K_{a} P_{L}+3 k \rho\left(T_{j}-T_{e}\right) / 2 m_{i} \tau_{e i}$,

$3 k \rho / 2 m_{i} D T_{j} / D t=-\rho p_{i} \cdot D(1 / \rho) / D t+3 k \rho\left(T_{e}-T_{i}\right) / 2 m_{i} \tau_{e j}$,

In these equations, the viscosity of the fluid and the bremsstrahlung are neglected. In the course of the implosion, the fusion reaction does not occur since the ion temperature is not so high. The independent variable $r, \theta, \phi$ and $t$ are transformed to

$$
r=\bar{r}+\tilde{r}(\bar{r}, \bar{\theta}, \bar{\phi}, \bar{t}), \quad \theta=\bar{\theta} \quad \phi=\bar{\phi} \quad t=\bar{t} .
$$

The dependent variables, for example $\rho$, are splitted into two parts,

$$
\rho_{-}(r, \theta, \phi, t)=\bar{p}(\bar{r}, \bar{t})+\tilde{p}(\bar{\rho}, \bar{\theta}, \bar{\phi}, \bar{t}) \text {, }
$$

where $\bar{\rho}$ is the value obtained in the one-dimensional motion, and $\tilde{\rho}$ is the perturbation accompanied by the digree of freedom of the three dimensions. After we linearize eq s. (13) - (16) with regard to the perturbed quantities, the perturbed quantities are expanded in the spherical harmonics. For example,

$$
\tilde{\rho}=\sum_{1, m} \hat{\rho}_{1 m}(\bar{r}, \bar{t}) \gamma_{1 m}(\bar{\theta}, \bar{\phi}) .
$$

Then eq. (13) reduces to where

$$
D \hat{\rho} / D t+\bar{\rho} \phi_{1 m}+\bar{\phi} \hat{\rho}_{1 m}=0 \text {, }
$$

$\Phi=\operatorname{div} v$.

We carry out numerical calculations for various 1. A result is shown in Fig.7. The perturbation grows considerably. More detailed inspections must be done for the stability of implosion.

\$4. Transport Coefficients

In $\S^{2}$, we use the classical values for the transport coefficients of the plasma. The numerical results. of course depend very much on the transport coefficients. In 
the classical forms, their values increase with the temperature. But in reality, the plasma is collisionless when the temperature is higher than $1 \mathrm{KeV}$.

Suppose a pellet of $r=500(\mu)$ radius. In the region $\Delta r=10(\mu)$ of the pellet surface, the laser energy of $E_{L}=10^{4}(\mathrm{~J})$ is assumed to be absorbed. The absorbed energy is shared with electrons in the high energy tail. If the internal energy Un of these electrons is assumed to arrive at

$$
U_{n_{1}}=m_{e} c^{2} / 2=4.1 \times 10^{-14}(\mathrm{~J}) \text {, }
$$

where $c$ is the light speed, then the total number $n_{h}$ of electrons with such a high internal energy is given by

$$
n_{h}=E_{L} / U_{n}=2.4 \times 10^{17} \text {. }
$$

Electrons which escape with the velocity c from the pellet surface where the density is critical, are pulled back through the electric field $E$ induced by the charge separation. The maximum length $\lambda$ in which the charge separation occurs can be estimated as follows,

$$
\lambda=c^{2} \mathrm{~m}_{\mathrm{e}} / 2 \mathrm{eE}=4 \pi \varepsilon c^{2} \mathrm{~m}_{\mathrm{e}} r / 2 \mathrm{e}^{2} \mathrm{n}_{h}=0.1(\mu) \text {. }
$$

This $\lambda$ is much smaller in comparison with, for example, the thickness of the density variation in Fig.2. It is inevitable to obtain the reasonable values of the transport coefficients in order to suggest the suitable experimental conditions for obtaining the maximum fusion energy in the pellet by a laser heating. For this purpose, we are starting to calculate the following Vlasov equations and Poisson equation ${ }^{15}$,

$$
\begin{aligned}
& \partial f_{i, e} / \partial t+u \partial f_{i, e} / \partial x \pm e E / m_{i, e} \cdot \partial f_{i, e} / \partial u=0, \\
& d E / d x=e \int_{-\infty}^{+\infty}\left(f_{i}-f_{e}\right) d u .
\end{aligned}
$$

At the initial time, the ion is homogeneous, and $E=0$ throughout the region. The electron has the low temperature in the negative $x$, and the electron has a high enerty tail in the positive $x$. The electron temperature $T_{e}$ versus the distance $x$ are plotted for several times in Fig.8. The wave lengthes seem to remain in a few Debye lengthes. More detailed investigations are also required to know the exact values of the transport coefficient, that is, to know the dissipation mechanisms.

\section{References}

1 A.F. Haught and D.H. Polk : Phys. of Fluids 13 (1970) 2825.

2 M.S. Chu : Phys: of Fruids 15 (1972) 413.

3 E.B. Goldman : Plasma Physics 15 (1973) 289.

4 J.W. Shearer and J.J. Duderstadt : Nuclear Fusion 13 (1973) 401.

5 J.S. Clarke, J.N. Fisher and R.J. Mason : Phys. Rev. Letters 30 (1973) 89.

6 C. Fauquignon and F. Floux : Phys. of Fluids 13 (1970) 386.

7 C. Yamanaka et al : IPPJ 117 (1972).

8 C. Yamanaka et al : Phys. Rev. A6 (1972) 2335.

9 R.D. Richtmyer and K.W. Morton : "Difference Methods for Initial-Value Problems" (Interscience, New York, 1957).

10 L. Spitzer.Jr. : "Physics of Füly Ionized Gasses" (Interscience, New York, 1961 ).

11 W.L. Kruer and J.M. Dawson : Phys. of Fluids 15 (1972) 446.

12 K. Nishikawa: J. Phys. Soc. Japan 24 (1968) $916,1152$.

13 A. Sakurai : J. Phys. Soc. Japan 36 (1974) 610.

14 J.N. Shiau, E.B. Goldman and C.I. Weng : Phys. Rev. Letters 32 (1974) 352.

$15 \mathrm{~J} . A$. Byers and J. Killeen : "Methods in Computational Physics" (Academic press, New York London, 1970) vol.9, p.265. 


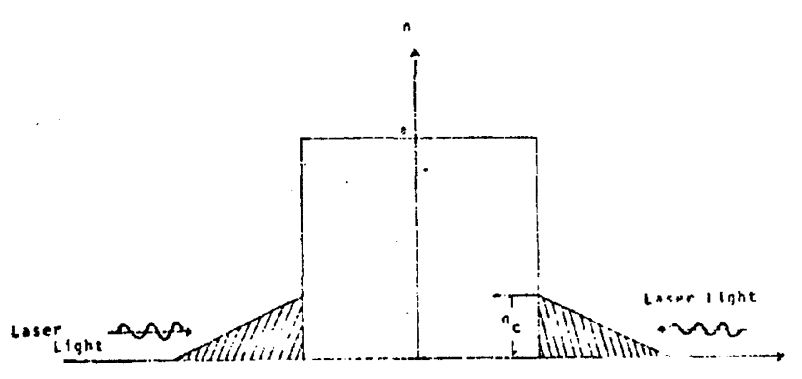

Fig. 1.

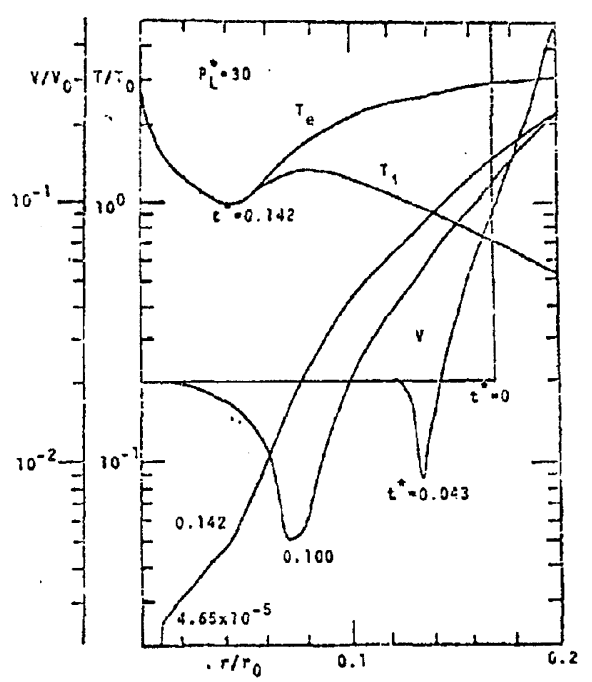

Fig. 2.

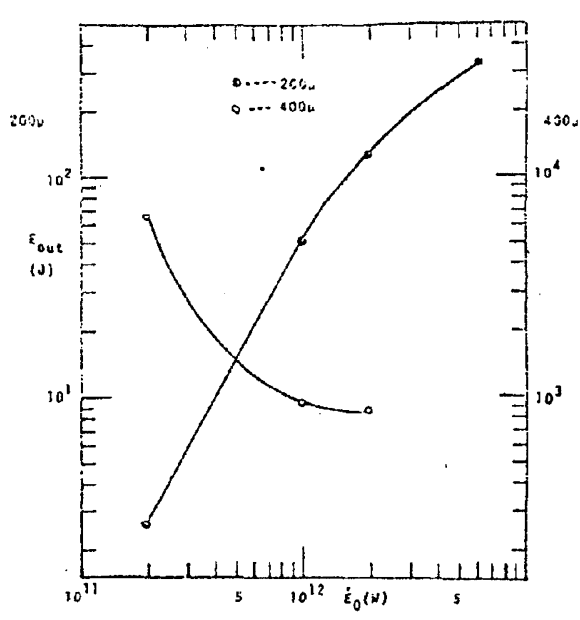

Fig. 4. 


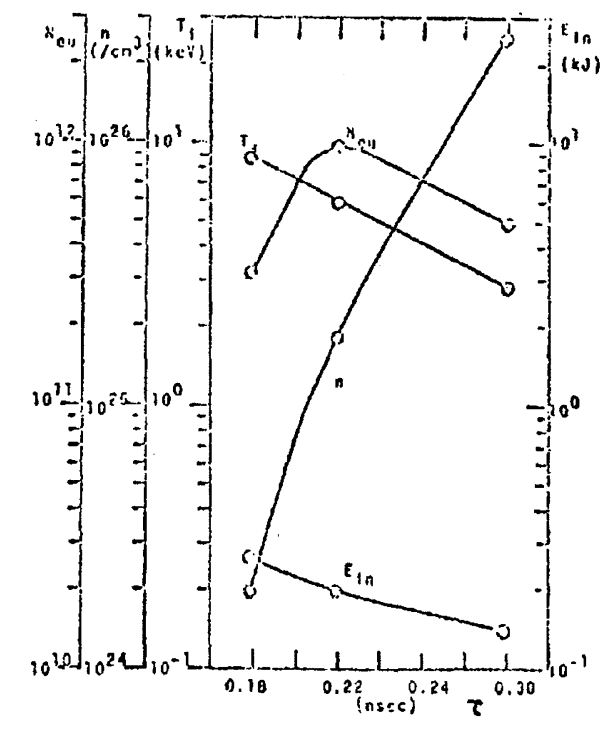

Fig. 5 .

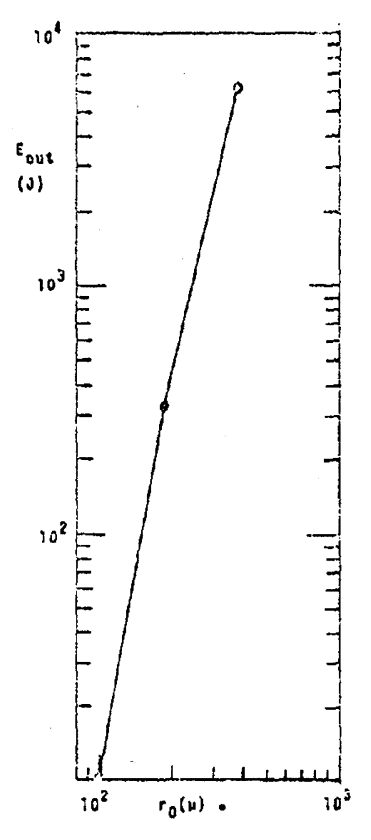

Fig. 6.

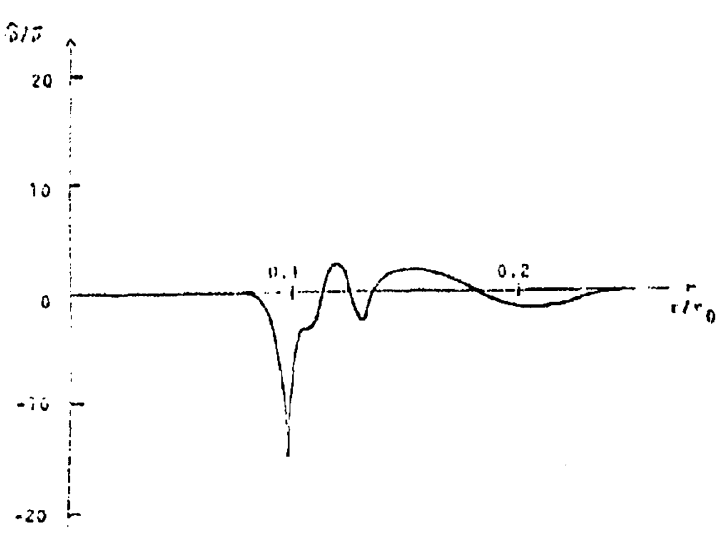

Fig. 7 .

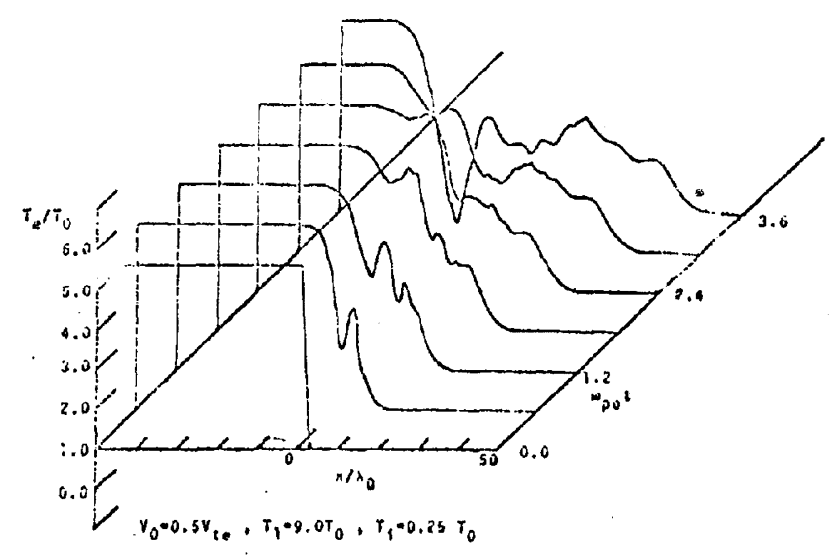

Fig. 3. 